\title{
Imidazole decreases the ampicillin resistance of an Escherichia coli strain isolated from a cow with mastitis by inhibiting the function of autoinducer 2
}

\author{
Lumin Yu, ${ }^{*}$ Wenchang Li, ${ }^{*}$ Ming Zhang, ${ }^{*}$ Yunmei Cui, $†$ Xiaolin Chen, ${ }^{*}$ Jingtian Ni, ${ }^{*} \mathrm{Li} \mathrm{Yu, \ddagger} \mathrm{Fei} \mathrm{Shang,}{ }^{* 1}$ \\ and Ting $X u e^{* 1}$ \\ *School of Life Sciences, and \\ †School of Sciences, Anhui Agricultural University, Hefei, Anhui 230036, China \\ fDepartment of Microbiology and Parasitology, Anhui Key Laboratory of Zoonoses, Anhui Medical University, Hefei 230032, China
}

\begin{abstract}
Extended-spectrum $\beta$-lactamase-positive Escherichia coli is an important causative agent of mastitis in dairy cows that results in reduced milk production and quality, and is responsible for severe economic losses in the dairy industry worldwide. The quorum sensing signaling molecule autoinducer 2 (AI-2) is produced by many species of gram-negative and gram-positive bacteria, and might be a universal language for intraspecies and interspecies communication. Our previous work confirmed that exogenous AI-2 increases the antibiotic resistance of extended-spectrum $\beta$-lactamase-positive $E$. coli to the $\beta$-lactam group of antibiotics by upregulating the expression of the TEM-type $\beta$-lactamase. In addition, this regulation relies on the function of the intracellular AI-2 receptor LsrR. In the present work, we reported that exogenous imidazole, a furan carbocyclic analog of AI-2, decreases the antibiotic resistance of a clinical E. coli strain to $\beta$-lactam antibiotics by inhibiting the function of AI-2.
\end{abstract}

Key words: autoinducer 2, Escherichia coli, mastitis, ampicillin resistance

\section{INTRODUCTION}

Mastitis is the most frequent disease in dairy cows and is responsible for severe economic losses in the dairy industry worldwide. It reduces milk production and causes poor milk quality, and represents a source of contamination of raw milk and dairy products. Mastitis is usually caused by bacterial pathogens including Staphylococcus aureus, Streptococcus uberis, Streptococcus dysgalactiae, Streptococcus agalactiae, Escherichia coli, and Klebsiella spp. (Riekerink et al., 2008; Mia et al., 2016). Previous studies have reported that E. coli is most commonly isolated pathogen from clinical

Received August 29, 2017.

Accepted December 13, 2017.

${ }^{1}$ Corresponding authors: shf@ahau.edu.cn and xuet@ahau.edu.cn mastitis (Kempf et al., 2016). Antimicrobial agents are widely used in livestock to control and prevent bovine mastitis. However, the abuse and misuse of antimicrobials including $\beta$-lactam antibiotics such as penicillin or ampicillin in livestock has contributed to the emergence of antimicrobial-resistant bacteria (Van Boeckel et al., 2015; Zhang et al., 2015; Mia et al., 2016). Some studies have reported the presence of extended spectrum $\beta$-lactamase (ESBL) genes in E. coli in slaughtered cattle and in milk from cows with mastitis (Hall et al., 2011; Reist et al., 2013; Timofte et al., 2014; Kuipers et al., 2016), and the emergence of the ESBL resistance mechanism presents a great challenge for the common use of $\beta$-lactam antibiotics and routine antimicrobial therapy (Jacoby and Medeiros, 1991; Eliopoulos and Bush, 2001; Tracy et al., 2001; Ahmed et al., 2007; Altayb et al., 2014; Xue et al., 2016). The TEM-type ESBL are recognized as one of the most prevalent mechanisms of resistance to $\beta$-lactam antibiotics in the Enterobacteriaceae (Pai et al., 2001; Baraniak et al., 2005). The multi-antibiotic resistance of TEM-type ESBL-producing E. coli is related to TEM gene located on large plasmid, which could transfer to other bacteria (Thomson and Amyes, 1993). Hence, TEM-type ESBLproducing bacteria pose a major problem for clinical therapeutics (Shiju et al., 2010).

Quorum sensing (QS) is a process of cell-to-cell communication in which bacteria use self-secreted chemical molecules known as autoinducers to regulate specific physiological functions in response to fluctuations in the population density in a community to better adapt to the external environment (Fuqua et al., 1994; Bassler, 1999, 2002; Li et al., 2006; Xue et al., 2009). When the concentration of autoinducers reaches a threshold, population-wide changes in gene expression are triggered, resulting in the modulation of a variety of physiological characteristics, such as antibiotic susceptibility and biofilm-forming ability (Bassler et al., 1994; Ahmer, 2004; Ahmed et al., 2007; Li et al., 2007; Xue et al., 2009). Autoinducer 2 (AI-2), which is produced by many species of gram-negative and gram-positive 
bacteria, is hypothesized to be part of a universal language for intraspecies and interspecies communication (Surette et al., 1999; Federle and Bassler, 2003; Sun et al., 2004). Studies have shown that dihydroxy-2,3pentanedione, which is a metabolite produced by the intracellularly activated methyl cycle, spontaneously cyclizes and then undergoes further rearrangements to form AI-2 signals. Extracellular AI-2 reaches a maximal concentration in the mid-exponential phase and is rapidly degraded in the stationary phase in E. coli (Winzer et al., 2003; Vendeville et al., 2005; Ahmed et al., 2007; Li et al., 2007).

In clinical antibiotic-resistant E. coli strains, whether or not AI-2 is related to antibiotic resistance gene expression has seldom been investigated (Thompson et al., 2015). Our one previous study reported that, in the presence of $\beta$-lactam groups of antibiotics, exogenous AI-2 increases the bacterial antibiotic resistance in an LsrR-dependent manner in strain ECDCM1, a clinical ESBL-positive E. coli strain was isolated from a dairy cow with mastitis (Xue et al., 2016). In the present study, we further investigated the effect of imidazole, a furan carbocyclic analog of AI-2, on the bacterial ampicillin resistance in strain ECDCM1, and our results indicated that imidazole decreases the bacterial resistance to ampicillin by inhibiting the regulatory effect of AI-2.

\section{MATERIALS AND METHODS}

\section{Bacterial Strains and Culture Conditions}

The E. coli strain used in this study was isolated from a dairy cow with mastitis. The $E$. coli was stored at $-80^{\circ} \mathrm{C}$. Before each experiment, it was first cultured on Luria broth agar plates that contained $10 \mathrm{~g} / \mathrm{L}$ of Bacto tryptone (Oxoid, Basingstoke, UK), $5 \mathrm{~g} / \mathrm{L}$ of yeast extract (Oxoid), $10 \mathrm{~g} / \mathrm{L}$ of NaCl (Sangon, Shanghai, China), and $20 \mathrm{~g} / \mathrm{L}$ of agar powder (Sangon) for 16 $\mathrm{h}$ at $37^{\circ} \mathrm{C}$ in air supplemented with $5 \% \mathrm{CO}_{2}$. Colonies were then cultivated overnight in $2 \mathrm{~mL}$ of MuellerHinton (MH) broth (Oxoid), designated as the first overnight cultures, and subsequently $E$. coli from the first overnight culture was diluted to an optical density at $600 \mathrm{~nm}$ of approximately 0.03 in fresh $\mathrm{MH}$ broth for the following experiments. Cultures of the E. coli strain were grown at $37^{\circ} \mathrm{C}$ with shaking at $200 \mathrm{rpm}$.

\section{Antimicrobial Activity Assay}

Antibacterial activity assays were performed according to the following methods. Colonies of strain ECDCM1 were placed into $3 \mathrm{~mL}$ of $\mathrm{MH}$ broth (Oxoid) and cultivated at $37^{\circ} \mathrm{C}$ with shaking at $180 \mathrm{rpm}$ for 20 h. The overnight cultures were inoculated into fresh $\mathrm{MH}$ broth and adjusted to an optical density at $600 \mathrm{~nm}$ of 0.03 before being dispensed into 96-well plates (Costar, Corning, Steuben, NY) containing serial dilutions of imidazole. Cells were incubated at $37^{\circ} \mathrm{C}$ for $16 \mathrm{~h}$, and 10 -fold serial dilutions of the cultures were performed by successive transfer $(0.1 \mathrm{~mL})$ through 6 microfuge tubes containing $0.9 \mathrm{~mL}$ of $\mathrm{MH}$. Subsequently, 100- $\mu \mathrm{L}$ dilutions were dropped onto $\mathrm{MH}$ agar plates. After culturing for $16 \mathrm{~h}$ at $37^{\circ} \mathrm{C}$, viable colonies were counted and the number of colony-forming units was compared between the control and test groups. The survival rates of the control groups with or without exposure to antibiotic were designated as $100 \%$. Experiments were repeated 3 times in 4 parallel experiments.

\section{Total RNA Isolation, cDNA Generation, and Real-Time PCR Processing}

The transcript levels of the antibiotic resistance gene $T E M$ and the AI-2 receptor LsrR coding gene lsrR were tested by performing real-time reverse-transcription (RT)-PCR experiments. First, overnight cultures of the clinical E. coli strain ECDCM1 were diluted 1:100 in $\mathrm{MH}$ broth in the presence of ampicillin and, when appropriate, AI-2 and imidazole were added to a final concentration of 39 and $200 \mu M$, respectively. The cultures were grown to the exponential phase at $37^{\circ} \mathrm{C}$ with shaking. Next, E. coli cells were collected by centrifugation and resuspended in Tris-EDTA buffer $(\mathrm{pH}$ 8.0) containing $10 \mathrm{~g} / \mathrm{L}$ of lysozyme. After incubation for $5 \mathrm{~min}$ at $37^{\circ} \mathrm{C}$, total RNA was extracted from the cells using Trizol reagent (Ambion, Austin, TX), and residual DNA was removed using DNaseI (TaKaRa, Dalian, China). Real-time RT-PCR was then performed using the PrimeScript 1st Strand cDNA synthesis kit, SYBR Premix Ex Taq (TaKaRa), and a StepOne realtime PCR system (Applied Biosystems, Carlsbad, CA). Last, differences in gene expression were calculated by the $\Delta \Delta \mathrm{Ct}$ (where $\mathrm{Ct}=$ cycle threshold) method, using the $16 S \mathrm{rDNA}$ gene as a housekeeping gene, normalized by subtracting the $\mathrm{Ct}$ value of $16 \mathrm{~S} \mathrm{cDNA}$ from that of the target cDNA. All of the real-time RT-PCR assays were repeated at least 3 times with similar results. The primers used in this study are listed in Table 1, and the PCR amplification efficiency was between 1.93 and 2.09 .

\section{Statistical Analysis}

All data were analyzed with the statistical software SPSS (ver. 19.0, IBM Corp., Armonk, NY) using a 1-way ANOVA method; the test results are shown as mean \pm standard deviation. Paired $t$-tests were used 
A

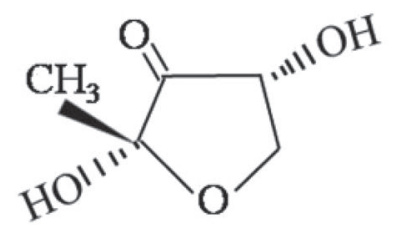

R-DHMF



S-DHMF

B<smiles>c1c[nH]cn1</smiles>

\section{Imidazole}

Figure 1. The chemical structural formulae of autoinducer 2 (AI-2) and imidazole: (A) the structural formulae of 2 different conformations of AI-2, and (B) the structural formulae of imidazole. S-THMF $=(2 S, 4 S)$-2-methyl-2,3,3,4-tetrahydroxytetrahydrofuran; R-THMF = (2R,4S)-2-methyl-2,3,3,4-tetrahydroxytetrahydrofuran.

for statistical comparisons between groups. The level of statistical significance was set at a $P$-value of $\leq 0.01$.

\section{RESULTS}

\section{Imidazole Decreases the Antibiotic Resistance of Strain ECDCM1 to Ampicillin}

To determine the influence of imidazole, which is also a furan carbocyclic analog (Figure 1), on $\beta$-lactam

Table 1. Oligonucleotide primers used in this study

\begin{tabular}{ll}
\hline Primer name & Oligonucleotide $\left(5^{\prime}-3^{\prime}\right)$ \\
\hline rt-16s-f & TTTGAGTTCCCGGCC \\
rt-16s-r & CGGCCGCAAGGTTAA \\
rt-lsrR-f & CGGATCGCGTGGTTTT \\
rt-lsrR-r & TCAACATATGCGCCGC \\
rt-TEM-f & AGAGTTTTCGCCCCG \\
rt-TEM-r & CCCCCATGTTGTGCA \\
\hline
\end{tabular}

antibiotic resistance of the E. coli strain ECDCM1, the survival rates of this strain were detected under different conditions. First, the survival rate of strain ECDCM1 was detected with the addition of different concentrations of imidazole in the absence of ampicillin. The survival rates of the test groups cultured with different concentrations of imidazole $(10,50,100,200$, and $400 \mu M$ ) were all similar to that of the control group without the addition of imidazole (Figure 2A). This result showed that imidazole does not affect the survival of strain ECDCM1 in the absence of ampicillin. Subsequently, we evaluated the effect of imidazole on survival of the strain ECDCM1 in the presence of ampicillin (the ampicillin concentration used in this experiment was 1/2 MIC). As shown in Figure 2B, in the presence of ampicillin, imidazole did not affect the survival rate of strain ECDCM1 at the concentrations of 10,50 , or $100 \mu M$; however, at the 200 and $400 \mu M$ concentrations, the survival rates of the strains were approximately 69 and $71 \%$, respectively, compared with the control group without imidazole. These data indicated that, in the presence of ampicillin, the addition of high concentrations of imidazole can decrease the resistance of strain ECDCM1 to the antibiotic.
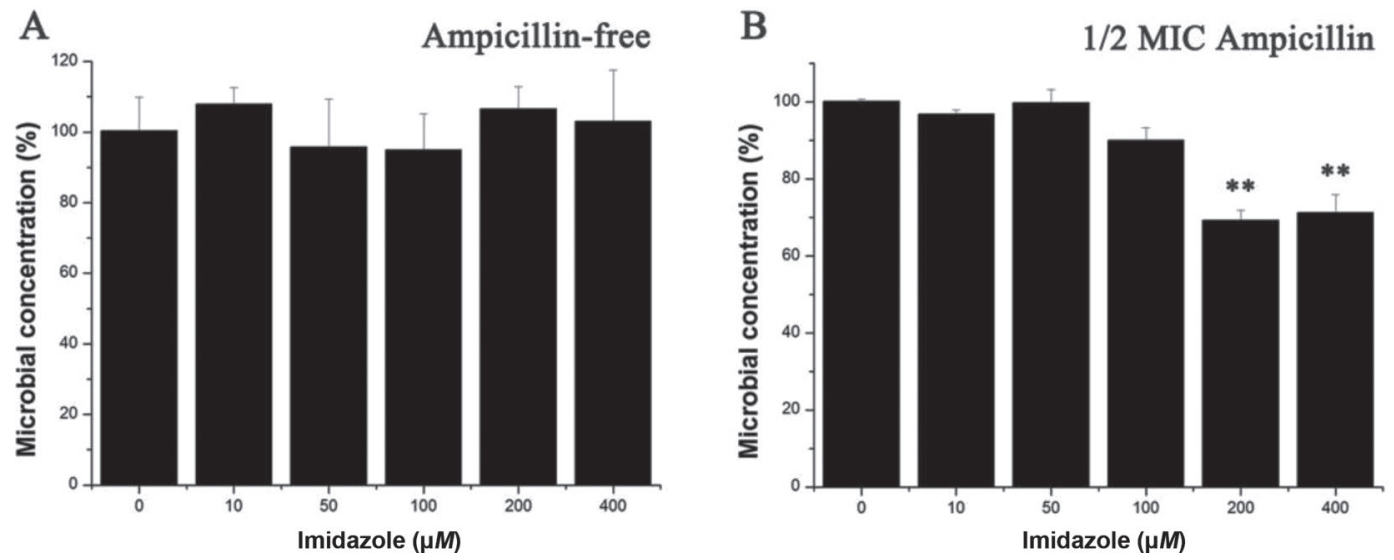

Figure 2. Colony-forming unit assays of Escherichia coli strain ECDCM1 incubated with different concentrations of imidazole in the absence or presence of ampicillin: (A) in the absence of ampicillin, and (B) in the presence of ampicillin. The survival rate of the control group without imidazole was designated $100 \%$. The colony counts of the test group cultured with imidazole at different concentrations were compared with those of the control group (without imidazole) in the absence or presence of ampicillin. Error bars indicate SD. The results represent a mean of 3 independent experiments; ${ }^{*} P<0.01$, indicating a difference between different imidazole concentrations. 


\section{Excess Imidazole Decreased the Resistance of Strain ECDCM1 to Ampicillin by Inhibiting the Function of Al-2}

The antibacterial assays were performed to examine whether imidazole affects the survival of strain ECDCM1 with the addition of exogenous AI-2. The results showed that, in the absence of ampicillin, the survival rates of the test groups with exogenous imidazole or AI-2 were similar to those of the control groups (Figure $3 \mathrm{~A})$; however, in the presence of ampicillin, the survival rate of the test group with AI-2 rose to $134 \%$ compared with that of the control group, in accordance with our previous report. Meanwhile, the survival rate of the test group with imidazole dropped to $69 \%$ compared with that of the control group, and the survival rate of the test group with exposure to imidazole and exogenous AI-2 dropped to $65 \%$ compared with that of the control group (Figure 3B).

\section{Excess Imidazole Downregulated the Transcription Levels of IsrR and TEM by Inhibiting the Function of Al-2}

To further confirm whether imidazole affects $\beta$-lactam antibiotic resistance in strain ECDCM1 through AI-2, the transcript levels of the genes TEM and $l s r R$ were measured with the addition of exogenous AI-2 or excess imidazole in the absence or presence of ampicillin. As shown in Figure 4B, in the presence of ampicillin, the addition of exogenous AI-2 resulted in the transcript levels of $l s r R$ and TEM increasing by 2.3 and 2.7 times, respectively, whereas the addition of excess imidazole led to a decrease in the transcript levels of $l s r R$ and TEM by 6.7 and 7.1 times, respectively. As expected, when AI-2 and imidazole were added at the same time, the transcript levels of $l s r R$ and TEM decreased to almost the same extent as when only imidazole was added (Figure 4B), indicating that the regulatory effect of AI-2 on $l s r R$ and TEM transcription was inhibited by imidazole. These results confirmed that a high concentration of imidazole decreases the ampicillin resistance of strain ECDCM1 by significantly downregulating the transcription of $l s r R$ and TEM through inhibition of the function of AI-2.

\section{Excess Imidazole Affected the Ampicillin Resistance of Strain ECDCM1 Only in the Exponential Phase}

According to previous studies, extracellular AI-2 activity peaks in the exponential phase, but drastically decreases upon entry into the stationary phase. To confirm whether the inhibitory effect of imidazole on AI-2 follows the same trend, antibacterial assays were performed in different growth phases. The results show that, in the presence of ampicillin, the survival rates of the test groups with exogenous imidazole or AI-2 were similar to those of the control groups in the early exponential phase and in the stationary phase (Figure 5A and Figure 5C); however, in the exponential phase, AI-2 increased the survival rate of strain ECDCM1 in the presence of ampicillin, and addition of imidazole inhibited this effect. These results provide further evidence that imidazole influences the ampicillin resistance of strain ECDCM1 via its inhibitory effect on AI-2.


Figure 3. Colony-forming unit assays of Escherichia coli strain ECDCM1 incubated in the absence or presence of ampicillin: (A) in the absence of ampicillin, and (B) in the presence of ampicillin. The survival rate of the control (Ctrl) group without imidazole or autoinducer 2 (AI-2) was designated $100 \%$. The colony counts of the test group cultured with $200 \mu M$ imidazole or $39 \mu M$ AI- 2 were compared with those of the control group (with ampicillin only), and the colony counts of the test group cultured with $200 \mu M$ imidazole and $39 \mu M$ AI- 2 were compared with those of another test group cultured with AI-2. Error bars indicate SD. The results represent a mean of 3 independent experiments; ${ }^{* *} P<$ 0.01, indicating a difference between control and AI-2, control and imidazole, as well as AI-2 and imidazole + AI-2. 


\section{DISCUSSION}

The production of ESBL is generally associated with resistance to the $\beta$-lactam group of antibiotics in the Enterobacteriaceae and the dissemination of strains of this species harboring ESBL in clinical settings is rising (Adler et al., 2012; Valentin et al., 2014). Hence the study of drug resistance regulation of ESBL is becoming more important. Previous study reported that tazobactam, which is a $\beta$-lactamase inhibitor, showed strong inhibitory activities against ESBL-producing strains (Ishii et al., 2001; Sader et al., 2014). It has previously been reported that synthetic antisense oligonucleotides efficiently inhibit gene expression of CTX-M-type ESBL in field and clinical isolates and restore sensitivity to the third-generation cephalosporin (Readman et al., 2016).
However, whether AI-2 and furan carbocyclic analogs of AI-2 affect bacterial $\beta$-lactam antibiotic resistance has not been reported. Our previous work reported that the addition of exogenous AI-2 increases the resistance of $E$. coli strains isolated from dairy cows to $\beta$-lactam antibiotics by upregulating the transcription of TEM (Xue et al., 2016). As a signaling molecule of the QS system in E. coli, extracellular AI-2 accumulates to a peak level in the late-exponential phase and subsequently exhibits its maximal regulatory activity. Referring to our previous results, this phenomenon indicates that in the presence of antibiotics, the AI-2 QS system makes the bacteria more resistant to antibiotics and increases the chance of survival after entering into the late-exponential phase. Therefore, in this study, we attempted to explore if any AI-2 analogs would interfere
A

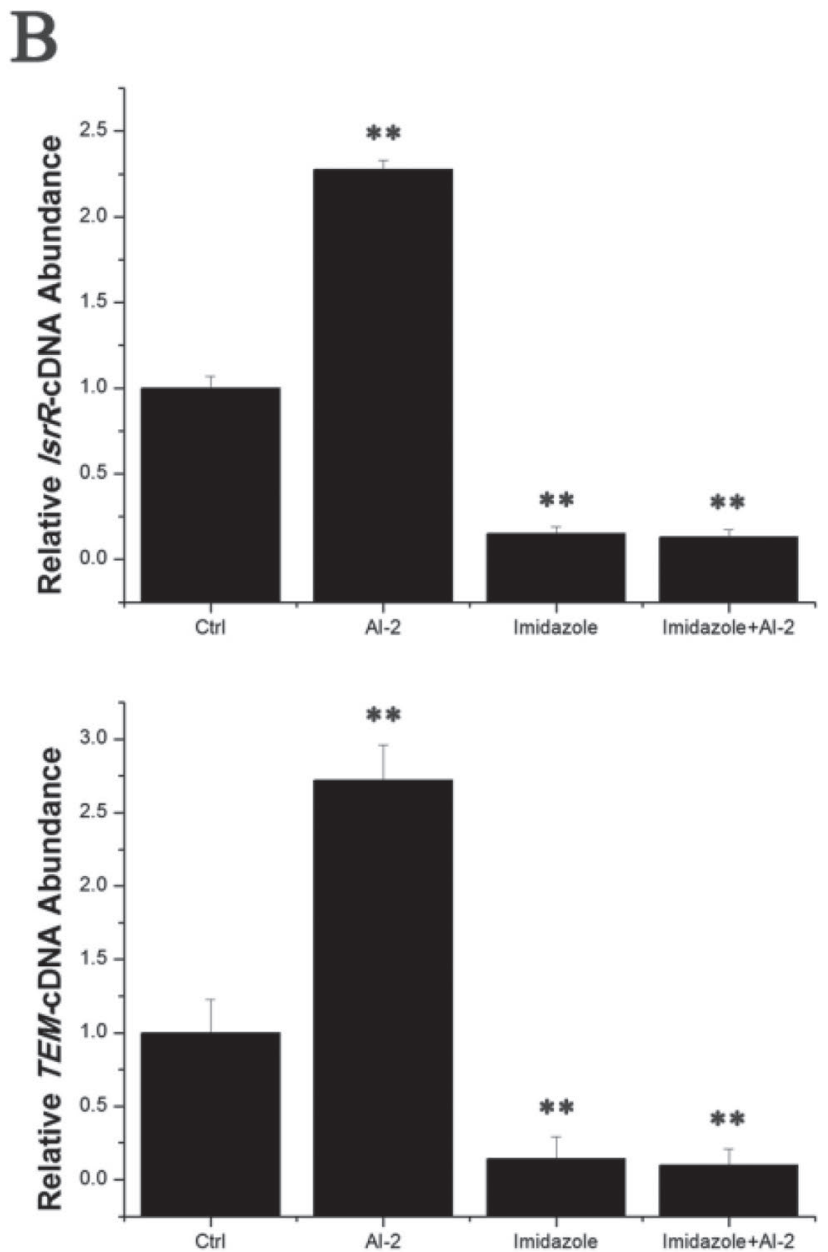

Figure 4. Comparative measurements of transcription (cDNA abundance) of lsrR and TEM in Escherichia coli strain ECDCM1 in the exponential phase. Relative transcript levels of $l s r R$ and $T E M$ were tested using real-time reverse-transcription-PCR in strain ECDCM1 cultured with $200 \mu M$ imidazole, $39 \mu M$ autoinducer 2 (AI-2), or $200 \mu M$ imidazole $+39 \mu M$ AI-2. (A) In the absence of ampicillin: (top) relative transcript levels of $l s r R$, and (bottom) relative transcript levels of TEM. (B) In the presence of ampicillin: (top) relative transcript levels of $l s r R$, and (bottom) relative transcript levels of TEM. Error bars indicate SD. The results represent a mean of 3 independent experiments; ** $P<0.01$, indicating a difference between control (Ctrl) and AI-2, control and imidazole, as well as AI-2 and imidazole + AI-2. 
A

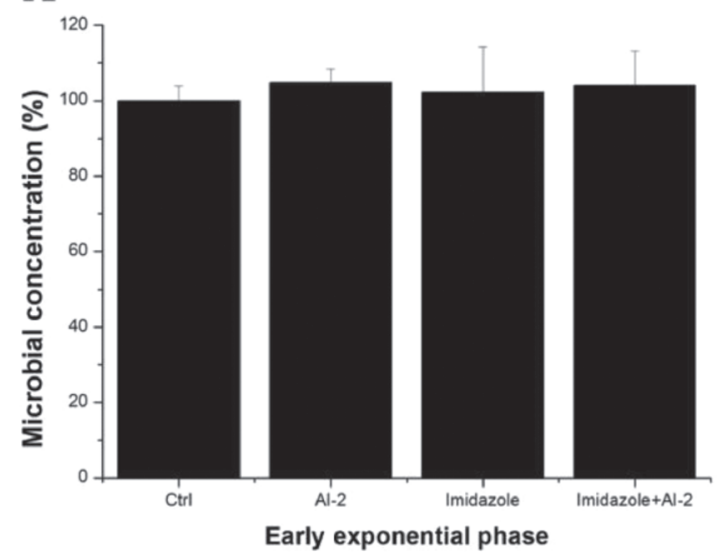

B

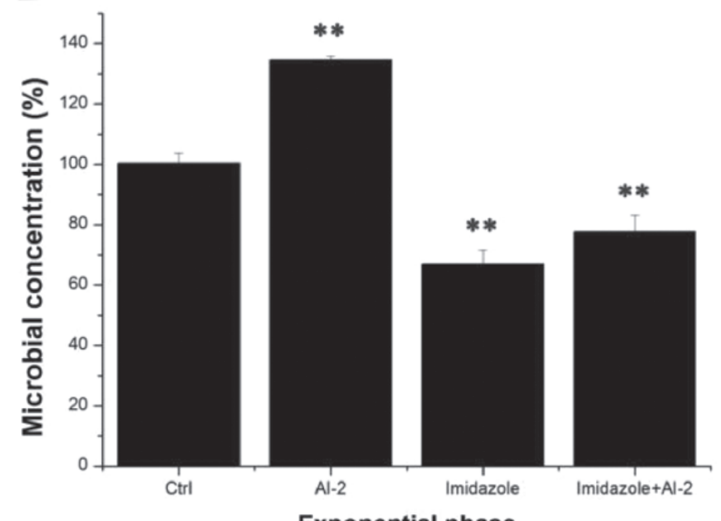

C

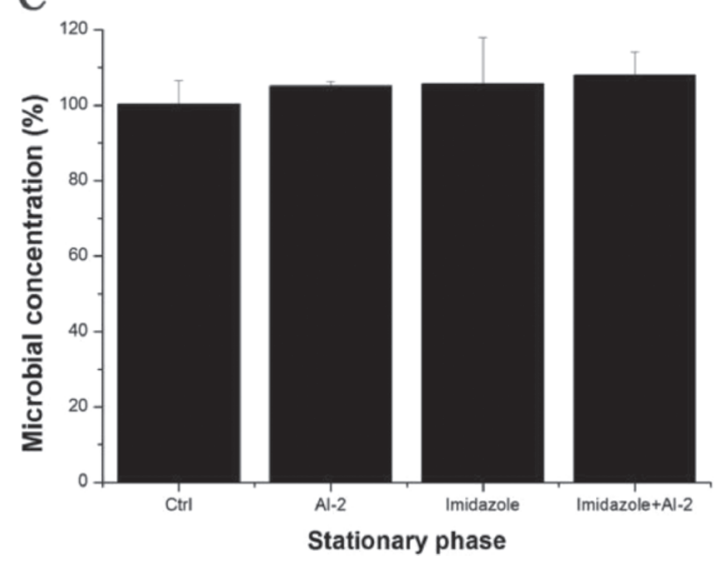

Figure 5. Colony-forming units assays of Escherichia coli strain ECDCM1 in the different growth phases. The survival rates of E. coli strain ECDCM1 cultured with $200 \mu M$ imidazole or $39 \mu M$ autoinducer 2 (AI-2) in the presence of ampicillin: (A) in the early exponential phase, (B) in the exponential phase, (C) in the stationary phase. The survival rate of the control (Ctrl) group without imidazole or AI-2 was designated $100 \%$. The colony counts of the test group cultured with imidazole or AI-2 were compared with those of the control group (with ampicillin only), and the colony counts of the test group cultured with imidazole and AI-2 were compared with those of another test group cultured with AI-2 in the presence of ampicillin. Error bars indicate $\mathrm{SD}$. The results represent a mean of 3 independent experiments; ${ }^{*} P$ $<0.01$, indicating a difference between control and AI-2, control and imidazole, as well as AI-2 and imidazole + AI-2. with the regulatory effect of AI-2 on bacterial antibiotic resistance, and our experiments indicate that imidazole has an apparent effect.

The results showed that in the presence of antibiotic (ampicillin), addition of excess imidazole inhibits the upregulating effect of AI-2 on antibiotic resistance, and even decreases antibiotic resistance, leading to a decline in the bacterial survival rate (Figure 3). In addition, real-time RT-PCR experiments confirmed that the transcript levels of TEM were not increased, but significantly decreased when excess imidazole and exogenous AI-2 were added at the same time. These results demonstrated that imidazole exerts its antibacterial activity by interfering with the activation effect of AI-2 on TEM transcription. Furthermore, we examined the effect of excess imidazole or exogenous AI-2 on bacterial antibiotic resistance in different growth phases. These results showed that the positive effect of AI-2 and the negative effect of imidazole are most apparent in the exponential phase.

In conclusion, we found that imidazole can downregulate the $\beta$-lactam antibiotic resistance of an E. coli strain isolated from dairy cows. This observation might provide potential new directions for studies focusing on the prevention and antibiotic treatment of infections caused by multidrug-resistant E. coli strains isolated from the dairy cows. In addition, the study was only carried out in a single E. coli isolated from dairy cows with mastitis and the experiments were only performed in vitro. Therefore, it is meaningful to explore the effect of imidazole (or other potential furan carbocyclic analogs) on antibiotic resistance in other isolates and measure the pharmarokinetics and pharmacodynamics of imidazole in vivo in future work.

\section{ACKNOWLEDGMENTS}

This work was supported by the National Natural Science Foundation of China (Beijing; grants 31672571 and 31371324).

\section{REFERENCES}

Adler, A., M. Gniadkowski, A. Baraniak, R. Izdebski, J. Fiett, W. Hryniewicz, S. Malhotra-Kumar, H. Goossens, C. Lammens, and Y. Lerman. 2012. Transmission dynamics of ESBL-producing Escherichia coli clones in rehabilitation wards at a tertiary care centre. Clin. Microbiol. Infection 18:E497-E505.

Ahmed, N. A. A. M., F. C. Petersen, and A. A. Scheie. 2007. AI-2 quorum sensing affects antibiotic susceptibility in Streptococcus anginosus. J. Antimicrob. Chemother. 60:49-53.

Ahmer, B. M. 2004. Cell-to-cell signalling in Escherichia coli and Salmonella enterica. Mol. Microbiol. 52:933-945.

Altayb, H. N., N. M. E. Amin, M. M. Mukhtar, M. A. Salih, and M. A. M. Siddig. 2014. Molecular characterization and in silico analysis of a novel mutation in TEM-1 beta-lactamase gene among 
pathogenic E. coli infecting a Sudanese patient. Am. J. Microbiol. Res. 2:217-223.

Baraniak, A., J. Fiett, A. Mrówka, J. Walory, W. Hryniewicz, and M. Gniadkowski. 2005. Evolution of TEM-type extended-spectrum B-lactamases in clinical Enterobacteriaceae strains in Poland. Antimicrob. Agents Chemother. 49:1872-1880.

Bassler, B. L. 1999. How bacteria talk to each other: Regulation of gene expression by quorum sensing. Curr. Opin. Microbiol. 2:582-587.

Bassler, B. L. 2002. Small talk. Cell-to-cell communication in bacteria Cell 109:421-424.

Bassler, B. L., M. Wright, and M. R. Silverman. 1994. Multiple signalling systems controlling expression of luminescence in Vibrio harveyi: Sequence and function of genes encoding a second sensory pathway. Mol. Microbiol. 13:273-286.

Eliopoulos, G. M., and K. Bush. 2001. New $\beta$-lactamases in gramnegative bacteria: Diversity and impact on the selection of antimicrobial therapy. Clin. Infect. Dis. 32:1085-1089.

Federle, M. J., and B. L. Bassler. 2003. Interspecies communication in bacteria. J. Clin. Invest. 112:1291-1299.

Fuqua, W. C., S. C. Winans, and E. P. Greenberg. 1994. Quorum sensing in bacteria: The LuxR-LuxI family of cell density-responsive transcriptional regulators. J. Bacteriol. 176:269-275.

Hall, L. V., C. M. Dierikx, J. C. Stuart, G. M. Voets, M. P. V. D. Munckhof, A. V. Essen-Zandbergen, T. Platteel, A. C. Fluit, N. V. D. Sande-Bruinsma, and J. Scharinga. 2011. Dutch patients, retail chicken meat and poultry share the same ESBL genes, plasmids and strains. Clin. Microbiol. Infect. 17:873-880.

Ishii, Y., J. Alba, M. Jun-ichi, and K. Yamaguchi. 2001. The effect of $\beta$-lactamase inhibitors against various extended-spectrum $\beta$-lactamase producers. Japanese J. Chemotherapy 49:562-564.

Jacoby, G. A., and A. A. Medeiros. 1991. More extended-spectrum beta-lactamases. Antimicrob. Agents Chemother. 35:1697-1704.

Kempf, F., C. Slugocki, S. E. Blum, G. Leitner, and P. Germon. 2016. Genomic comparative study of bovine mastitis Escherichia coli. PLoS One 11:e0147954.

Kuipers, A., W. J. Koops, and H. Wemmenhove. 2016. Antibiotic use in dairy herds in the Netherlands from 2005 to 2012. J. Dairy Sci. 99:1632-1648

Li, J., C. Attila, L. Wang, T. K. Wood, J. J. Valdes, and W. E. Bentley. 2007. Quorum sensing in Escherichia coli is signaled by AI-2/ LsrR: Effects on small RNA and biofilm architecture. J. Bacteriol. 189:6011-6020.

Li, J., L. Wang, Y. Hashimoto, C. Y. Tsao, T. K. Wood, J. J. Valdes, E. Zafiriou, and W. E. Bentley. 2006. A stochastic model of Escherichia coli AI-2 quorum signal circuit reveals alternative synthesis pathways. Mol. Syst. Biol. 2:67.

Mia, M. T., M. K. Hossain, N. A. Rumi, M. S. Rahman, M. S Mahmud, and M. Das. 2016. Detection of bacterial species from clinical mastitis in dairy cows at Nilphamari district and their antibiogram studies. Asian J. Med. Biol. Res. 2:656-663.

Pai, H., H. J. Lee, E. H. Choi, J. Kim, and G. A. Jacoby. 2001. Evolution of TEM-related extended-spectrum beta-lactamases in Korea. Antimicrob. Agents Chemother. 45:3651-3653.

Readman, J. B., G. Dickson, and N. G. Coldham. 2016. Translational inhibition of CTX-M extended spectrum beta-lactamase in clinical strains of Escherichia coli by synthetic antisense oligonucleotides partially restores sensitivity to cefotaxime. Front. Microbiol. 7:373.

Reist, M., N. Geser, H. Hächler, S. Schärrer, and R. Stephan. 2013. ESBL-producing Enterobacteriaceae: Occurrence, risk factors for fecal carriage and strain traits in the Swiss slaughter cattle population younger than 2 years sampled at abattoir level. PLoS One 8:e71725.

Riekerink, R. G. M. O., H. W. Barkema, D. F. Kelton, and D. T. Scholl. 2008. Incidence rate of clinical mastitis on Canadian dairy farms. J. Dairy Sci. 91:1366-1377.
Sader, H. S., P. R. Rhomberg, and R. N. Jones. 2014. Post $\beta$-lactamase inhibitor effect of Tazobactam in combination with Ceftolozane and tested against ESBL-producing strains. Antimicrob. Agents Chemother. 58:2434-2437.

Shiju, M. P., R. Yashavanth, and N. Narendra. 2010. Detection of extended spectrum beta-lactamase production and multidrug resistance in clinical isolates of E. coli and K. pneumoniae in Mangalore. J. Clin. Diagn. Res. 4:2442-2445.

Sun, J., R. Daniel, I. Wagner-Döbler, and A.-P. Zeng. 2004. Is autoinducer-2 a universal signal for interspecies communication: A comparative genomic and phylogenetic analysis of the synthesis and signal transduction pathways. BMC Evol. Biol. 4:36. https:// doi.org/10.1186/1471-2148-4-36.

Surette, M. G., M. B. Miller, and B. L. Bassler. 1999. Quorum sensing in Escherichia coli, Salmonella typhimurium, and Vibrio harveyi: A new family of genes responsible for autoinducer production. Proc. Natl. Acad. Sci. USA 96:1639-1644.

Thompson, J. A., R. A. Oliveira, A. Djukovic, C. Ubeda, and K. B. Xavier. 2015. Manipulation of the quorum sensing signal AI-2 affects the antibiotic-treated gut microbiota. Cell Reports 10:18611871

Thomson, C. J., and S. G. Amyes. 1993. Molecular epidemiology of the plasmid-encoded TEM-1 beta-lactamase in Scotland. Epidemiol. Infect. 110:117-125.

Timofte, D., I. E. Maciuca, N. J. Evans, H. Williams, A. Wattret, J. C. Fick, and N. J. Williams. 2014. Detection and molecular characterization of Escherichia coli CTX-M-15 and Klebsiella pneumoniae SHV-12 $\beta$-lactamases from bovine mastitis isolates in the United Kingdom. Antimicrob. Agents Chemother. 58:789-794.

Tracy, M., A. Wanahita, Y. Shuhatovich, E. A. Goldsmith, J. E. C. Iii, and D. M. Musher. 2001. Antibiotic susceptibilities of genetically characterized Streptococcus milleri group strains. Antimicrob. Agents Chemother. 45:1511-1514.

Valentin, L., H. Sharp, K. Hille, U. Seibt, J. Fischer, Y. Pfeifer, G. B. Michael, S. Nickel, J. Schmiedel, and L. Falgenhauer. 2014 Subgrouping of ESBL-producing Escherichia coli from animal and human sources: An approach to quantify the distribution of ESBL types between different reservoirs. Int. J. Med. Microbiol. 304:805-816.

Van Boeckel, T. P., C. Brower, M. Gilbert, B. T. Grenfell, S. A. Levin, T. P. Robinson, A. Teillant, and R. Laxminarayan. 2015. Global trends in antimicrobial use in food animals. Proc. Natl. Acad. Sci. USA 112:5649-5654.

Vendeville, A., K. Winzer, K. Heurlier, C. M. Tang, and K. R. Hardie. 2005. Making 'sense' of metabolism: Autoinducer-2, LuxS and pathogenic bacteria. Nat. Rev. Microbiol. 3:383-396.

Winzer, K., K. R. Hardie, and P. Williams. 2003. LuxS and autoinducer-2: Their contribution to quorum sensing and metabolism in bacteria. Adv. Appl. Microbiol. 53:291-396.

Xue, T., H. Sun, X. Zhou, L. Zhao, and B. Sun. 2009. LsrR-binding site recognition and regulatory characteristics in Escherichia coli AI-2 quorum sensing. Cell Res. 19:1258-1268.

Xue, T., L. Yu, F. Shang, W. Li, M. Zhang, J. Ni, and X. Chen. 2016. Short communication: The role of autoinducer 2 (AI-2) on antibiotic resistance regulation in an Escherichia coli strain isolated from a dairy cow with mastitis. J. Dairy Sci. 99:4693-4698.

Zhang, Q. Q., G. G. Ying, C. G. Pan, Y. S. Liu, and J. L. Zhao. 2015. Comprehensive evaluation of antibiotics emission and fate in the river basins of China: Source analysis, multimedia modeling, and linkage to bacterial resistance. Environ. Sci. Technol. 49:6772-6782 